ARTICLE

https://doi.org/10.1038/s41467-018-08223-5

\title{
Discovery of TaFeSb-based half-Heuslers with high thermoelectric performance
}

\author{
Hangtian Zhu (10 1, Jun Mao (D) 1, Yuwei Li ${ }^{2}$, Jifeng Sun², Yumei Wang ${ }^{3}$, Qing Zhu', Guannan Li ${ }^{4}$, Qichen Song (D) ${ }^{5}$, \\ Jiawei Zhou ${ }^{5}$, Yuhao $\mathrm{Fu}^{2}$, Ran He ${ }^{6}$, Tian Tong7${ }^{7}$, Zihang Liu', Wuyang Ren ${ }^{1,8}$, Li You ${ }^{1,9}$, Zhiming Wang ${ }^{8}$, \\ Jun Luo (10) ${ }^{9}$, Andrei Sotnikov ${ }^{6}$, Jiming Bao (10 ${ }^{7}$, Kornelius Nielsch ${ }^{6}$, Gang Chen (1) ${ }^{5}$, David J. Singh ${ }^{2}$ \& \\ Zhifeng $\operatorname{Ren}^{1}$
}

Discovery of thermoelectric materials has long been realized by the Edisonian trial and error approach. However, recent progress in theoretical calculations, including the ability to predict structures of unknown phases along with their thermodynamic stability and functional properties, has enabled the so-called inverse design approach. Compared to the traditional materials discovery, the inverse design approach has the potential to substantially reduce the experimental efforts needed to identify promising compounds with target functionalities. By adopting this approach, here we have discovered several unreported half-Heusler compounds. Among them, the p-type TaFeSb-based half-Heusler demonstrates a record high $Z T$ of $\sim 1.52$ at $973 \mathrm{~K}$. Additionally, an ultrahigh average $Z T$ of $\sim 0.93$ between 300 and $973 \mathrm{~K}$ is achieved. Such an extraordinary thermoelectric performance is further verified by the heatto-electricity conversion efficiency measurement and a high efficiency of $\sim 11.4 \%$ is obtained. Our work demonstrates that the TaFeSb-based half-Heuslers are highly promising for thermoelectric power generation.

\footnotetext{
${ }^{1}$ Department of Physics and Texas Center for Superconductivity, University of Houston, Houston, TX 77204, USA. ${ }^{2}$ Department of Physics and Astronomy, University of Missouri, Columbia, MO 65211, USA. ${ }^{3}$ Beijing National Laboratory for Condensed Matter Physics, Institute of Physics, Chinese Academy of Sciences, P.O. Box 603Beijing 100190, China. ${ }^{4}$ Department of Materials and Energy, Southwest University, Chongqing 400715, China. ${ }^{5}$ Department of Mechanical Engineering, Massachusetts Institute of Technology, Cambridge, MA 02139, USA. ${ }^{6}$ Institute for Metallic Materials, IFW-Dresden, Dresden 01069, Germany. ${ }^{7}$ Department of Electrical and Computer Engineering, University of Houston, Houston, TX 77204 , USA. ${ }^{8}$ Institute of Fundamental and Frontier Sciences, University of Electronic Science and Technology of China, Chengdu 610054, China. ${ }^{9}$ School of Materials Science and Engineering, Shanghai University, Shanghai 200444, China. These authors contributed equally: Hangtian Zhu, Jun Mao. Correspondence and requests for materials should be addressed to D.J.S. (email: singhdj@missouri.edu) or to zren@uh.edu)
} 
T he ever-increasing energy consumption from fossil-fuel combustion has led to alarming environmental impacts. As one of the clean energy conversion techniques, thermoelectric power generation can harvest waste heat and convert it into electricity via the Seebeck effect ${ }^{1,2}$. However, the wide application of thermoelectric power generation systems requires significant improvements in the energy conversion efficiency, which essentially depends on the materials' dimensionless figureof-merit $(Z T)$. It is defined as $Z T=S \sigma T /\left(\kappa_{\text {lat }}+\kappa_{\text {ele }}\right)$, where $S$ is the Seebeck coefficient, $\sigma$ is the electrical conductivity, $\kappa_{\text {lat }}$ is the lattice thermal conductivity, $\kappa_{\text {ele }}$ is the electronic thermal conductivity, and $T$ is the absolute temperature ${ }^{3-7}$.

The transport parameters on which $Z T$ depends are strongly interrelated with each other due to their different, and typically opposite, dependencies on carrier concentration and electronic structure $^{8}$. Therefore, research on thermoelectric materials has focused on identifying approaches that can effectively decouple the key transport parameters for enhancing the $Z T$ of existing materials ${ }^{9-14}$. In the meantime, relentless efforts have also been devoted to discovering promising new compounds that have unusual characteristics enabling favorable combinations of high electrical conductivity, large Seebeck coefficient, and low thermal conductivity. Historically, the materials discovery has mostly relied on the Edisonian trial and error approach. However, such a traditional process becomes increasingly time-consuming. As a result, there is a clear need for more efficient rational approaches to discovering new promising materials. This has provided the motivation for high-throughput methods and other theoretical approaches that use predictions of properties to identify candidate materials ${ }^{15,16}$. Yet a key challenge is to balance the reliability and cost in such studies. A consequence of the low reliability is that the predicted compounds (especially ones that have not yet been experimentally made) with promising thermoelectric performance may be experimentally identified as metastable or unstable. This is a particular challenge for thermoelectrics because a main approach for resolving the opposite dependencies of the transport properties, e.g., $S$ and $\sigma$, is through unusual electronic structures, but unusual electronic properties are often found in calculations for unstable compounds, e.g., due to unfavorable bonding. Thus, mere prediction of the thermoelectric properties for the missing compounds is not enough to guarantee experimental success. In fact, analysis of the thermodynamic stability for the unreported compounds and assessment of potential unknown competing phases could provide highly valuable guidance for the experimental efforts. It should be noted that careful experimental synthesis and evaluation of a compound are costly, while most theoretical calculations, especially as applied in high throughput modes, are relatively inexpensive. As such, it might be beneficial to use more sophisticated theoretical studies in predicting compounds before devoting the efforts for careful experimental study.

Recently, Gautier et al. ${ }^{17}$ investigated the thermodynamic stability of 400 unreported half-Heusler compounds and 54 of them were predicted to be stable. Subsequently, 15 compounds were successfully synthesized to verify their prediction. Similarly, Carrete et al. ${ }^{18}$ performed the thermodynamic analysis for 450 half-Heusler compounds and predicted that 77 are stable. In addition, Zakutayev et al. ${ }^{19}$ investigated the thermodynamic stability of the V-IX-IV family $(\mathrm{V}=\mathrm{V}, \mathrm{Nb}$, and $\mathrm{Ta}$; IX $=\mathrm{Co}$, Rh, and $\mathrm{Ir}$; and $\mathrm{IV}=\mathrm{C}, \mathrm{Si}, \mathrm{Ge}, \mathrm{Sn}$, and $\mathrm{Pb}$ ) of half-Heuslers. Their calculations indicated that among the 29 missing compounds, 8 are stable. TaCoSn, one of those predicted to be stable, was later successfully synthesized, confirming the prediction. Clearly, the inverse design approach ${ }^{20}$, which targets at the specific functionalities and involves with calculations of structure as well as thermodynamic stability and then followed by the experimental realization, is capable of significantly expediting the material discovery.

In our work, we focused on the half-Heusler compounds, which are among the most promising candidates for thermoelectric applications. Thermodynamic stabilities of the $\mathrm{V}^{1}$-VIII$\mathrm{V}^{2}$ family (with $\mathrm{V}^{1}=\mathrm{V}, \mathrm{Nb}$, and $\mathrm{Ta}$; $\mathrm{VIII}=\mathrm{Fe}, \mathrm{Ru}$, and $\mathrm{Os}$; and $\mathrm{V}^{2}=\mathrm{As}, \mathrm{Sb}$, and $\mathrm{Bi}$ ) of half-Heuslers were systematically investigated. As a result, we have discovered 6 undocumented compounds and 5 of them are stable with the half-Heusler crystal structure. The p-type TaFeSb-based half-Heusler, one of the compounds discovered in this work, demonstrated a very promising thermoelectric performance. A peak $Z T$ of $\sim 1.52$ at $973 \mathrm{~K}$ and an average $Z T$ of $\sim 0.93$ in the temperature range of 300 973 $\mathrm{K}$ were obtained. The high thermoelectric performance was further verified by the heat-to-electricity conversion efficiency measurement. A record high conversion efficiency of $\sim 11.4 \%$ at the cold-side temperature of $317 \mathrm{~K}$ and hot-side temperature of $973 \mathrm{~K}$ was obtained.

\section{Results}

Thermodynamic stability analysis. Systematic theoretical calculations on the $\mathrm{V}^{1}$-VIII- $\mathrm{V}^{2}$ family (with $\mathrm{V}^{1}=\mathrm{V}, \mathrm{Nb}$, and $\mathrm{Ta}$; VIII $=\mathrm{Fe}, \mathrm{Ru}$, and $\mathrm{Os}$; and $\mathrm{V}^{2}=\mathrm{As}, \mathrm{Sb}$, and $\mathrm{Bi}$ ) of halfHeusler phases were first conducted. In this family (27 compounds in total), 8 compounds have been documented to be stable while no experimental reports can be found for the remaining 19. Therefore, the crystal structure search for the missing compounds was conducted and the thermodynamic stability analysis of each compound with respect to all of the other competing phases was performed. The key aspect of the crystal structure search is that it allows us to check whether the half-Heusler structure is the true ground state rather than other possibly competing structures with the same 1:1:1 stoichiometry. We predict that 6 compounds (VRuAs, NbRuAs, TaRuAs, TaFeSb, NbOsSb, and TaOsSb) out of the missing 19 are stable, as shown in Fig. 1. All of the predicted stable compounds are in the cubic structure $(F 43 m)$, with the exception of NbRuAs that has an orthorhombic structure (Pnma). Some of these structures predicted agree quite well with the other theoretical calculations ${ }^{18,21}$. It should be noted that our prediction for NbRuAs differs from that of Carrete et al. ${ }^{18}$. We verified that the orthorhombic structure we found from the crystal structure prediction does have lower energy than the cubic structure they identified. The predicted energy difference is $25 \mathrm{meV}$ per atom. In the following, we will focus mainly on TaFeSb, and the other five stable compounds will be reported elsewhere.

For the thermodynamic stability of the $\mathrm{V}^{1}-\mathrm{VIII}-\mathrm{V}^{2}$ family, three criteria should be satisfied

$$
\begin{gathered}
\Delta \mu_{\mathrm{V}^{1}}+\Delta \mu_{\mathrm{VIII}}+\Delta \mu_{\mathrm{V}^{2}}=\Delta H_{f}\left(\mathrm{~V}^{1} \mathrm{VIIIV}^{2}\right) ; \\
\Delta \mu_{i}<0,\left(i=\mathrm{V}^{1}, \mathrm{VIII}, \mathrm{V}^{2}\right) ;
\end{gathered}
$$

and

$$
\begin{gathered}
n_{j} \Delta \mu_{\mathrm{V}^{1}}+m_{j} \Delta \mu_{\mathrm{VIII}}+q_{j} \Delta \mu_{\mathrm{V}^{2}} \leq \Delta H_{f}\left(\mathrm{~V}_{n_{j}}^{1} \mathrm{VIII}_{m_{j}} \mathrm{~V}_{q_{j}}^{2}\right), \\
j=1, \ldots, t,
\end{gathered}
$$

where $\Delta \mu_{i}=\mu_{i}-\mu_{i}^{0}$ is the deviation of actual chemical potential of atomic species $i$ during growth $\left(\mu_{i}\right)$ from that of bulk elemental solid or gas phase $\left(\mu_{i}^{0}\right), \Delta H_{f}$ is the heat of formation, and $\mathrm{V}_{n_{i}}^{1} \mathrm{VIII}_{m_{j}} \mathrm{~V}_{q_{i}}^{2}$ represents all of the known $j$ competing phases, which provide a limitation to the broadening of the stable regions. Eq. (1) is for equilibrium growth, Eq. (2) is to prevent the precipitation of elemental phases of atomic 
a

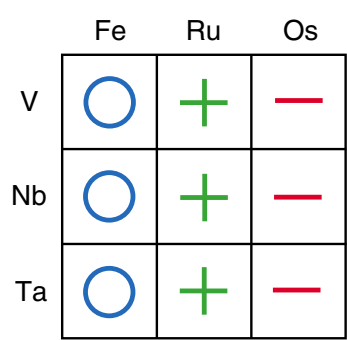

b

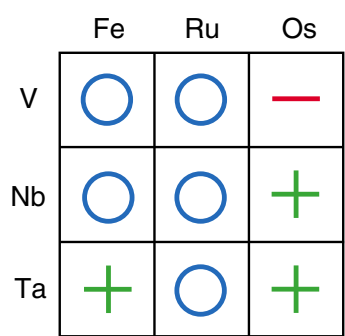

C

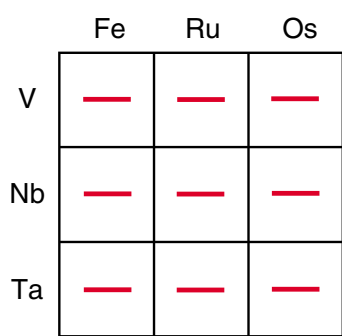

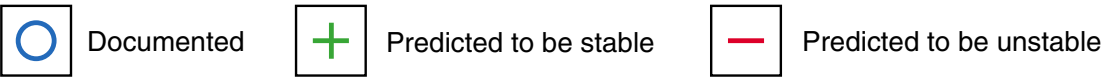

Fig. 1 Thermodynamic stability calculation. Calculated stability of the $\mathrm{V}^{1}-\mathrm{VIII}-\mathrm{V}^{2}$ family (with $\mathrm{V}^{1}=\mathrm{V}, \mathrm{Nb}$, and $\mathrm{Ta}$; $\mathrm{VIII}=\mathrm{Fe}, \mathrm{Ru}$, and $\mathrm{Os}$; and $\mathrm{V}^{2}=\mathrm{As}$, $\mathrm{Sb}$, and $\mathrm{Bi}$ ) of half-Heusler compounds. a $\mathrm{V}^{1}$-VIII-As, b $\mathrm{V}^{1}$-VIII-Sb, and $\mathbf{c} \mathrm{V}^{1}-\mathrm{VIII}-\mathrm{Bi}$

a

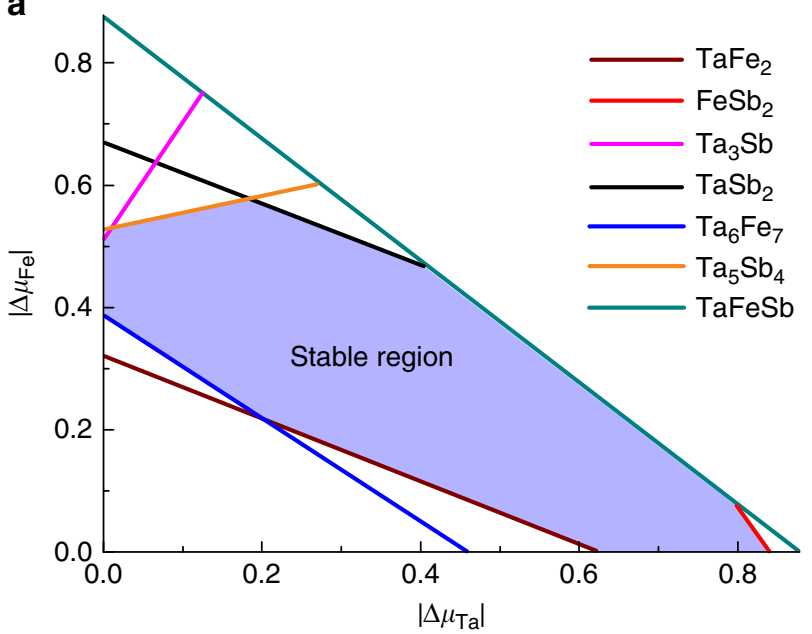

C

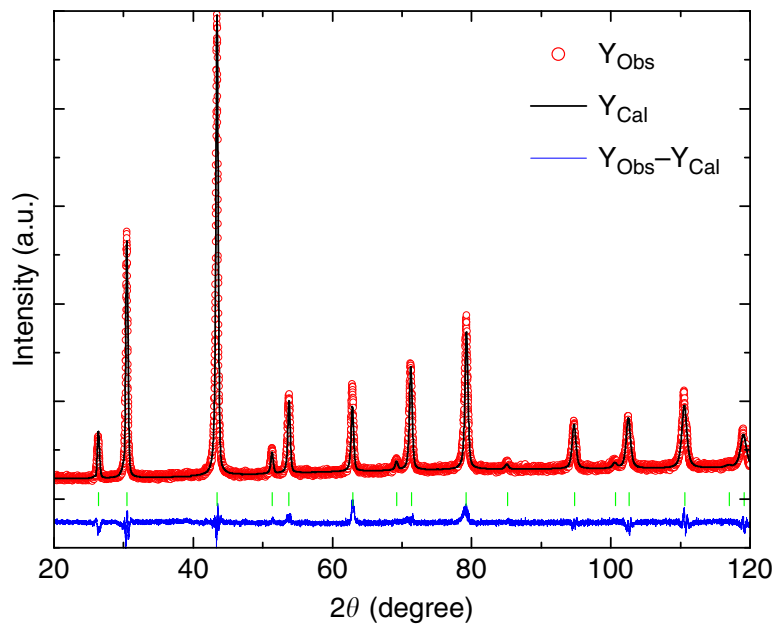

b

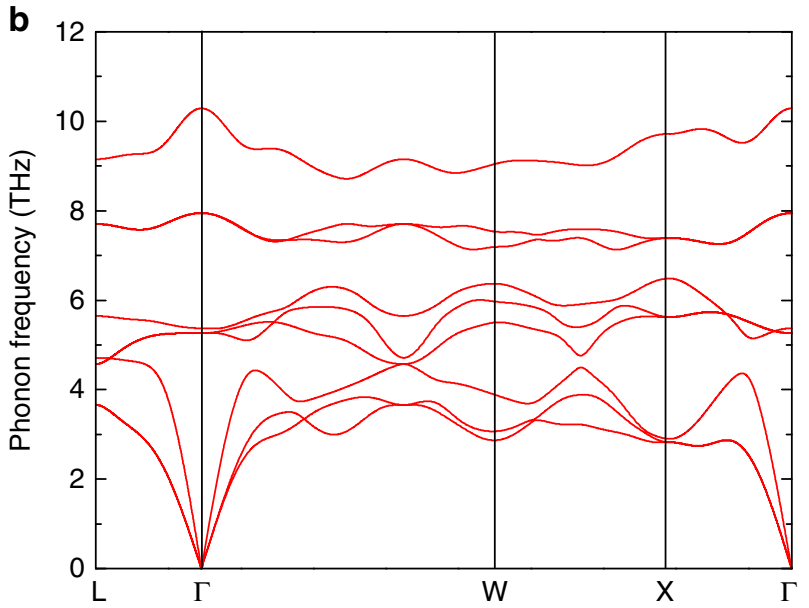

d

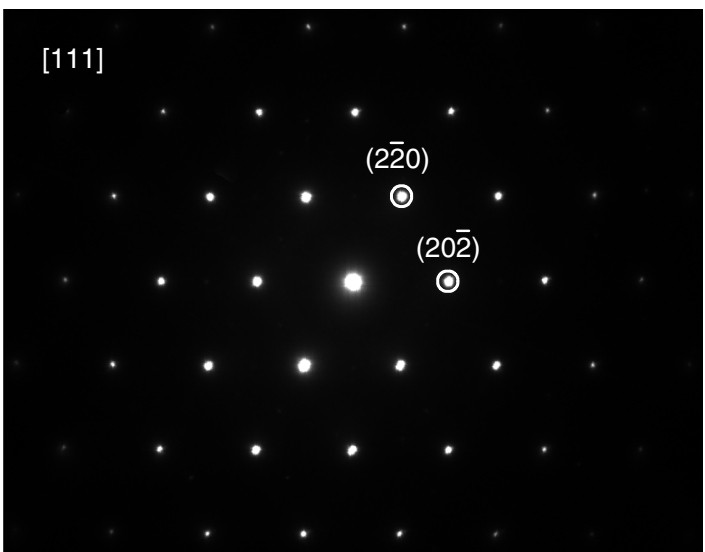

Fig. 2 Theoretical prediction and experimental realization of TaFeSb half-Heusler. a Phase stability diagram for TaFeSb, where each line represents a known competing phase and the stable region is indicated in violet. It should be noted that $\Delta \mu_{\mathrm{Fe}}$ and $\Delta \mu_{\mathrm{Ta}}$ are both negative. b) Calculated phonon dispersion of TaFeSb. c Rietveld refinement for the XRD pattern of the prepared TaFeSb in this work. $\mathbf{d}$ The [111] zone-axis selected area electron diffraction pattern

species $i$, and Eq. (3) is to ensure that $\mathrm{V}^{1}$-VIII-V $\mathrm{V}^{2}$ half-Heuslers are stable against the formation of other competing phases. Figure 2a shows the two-dimensional phase stability diagram for TaFeSb with two independent quantities $\Delta \mu_{\mathrm{Fe}}$ and $\Delta \mu_{\mathrm{Ta}}$. Six competing phases in the $\mathrm{Ta}-\mathrm{Fe}-\mathrm{Sb}$ system have been calculated to evaluate the stability of $\mathrm{TaFeSb}^{22,23}$. It should be noted that the $\mathrm{Fe}-\mathrm{Sb}$ phase diagram includes a $\mathrm{Fe}_{1.27} \mathrm{Sb}$ phase. This phase is characterized by site disorder, and thus was not included in our calculations. A stable region for TaFeSb (marked by violet) exists with the consideration of all known potential competing phases. Such a large stability region for $\mathrm{TaFeSb}$ indicates that this compound should be synthesized experimentally. In addition, 
the lattice dynamical stability of TaFeSb was also examined and its phonon dispersion is shown in Fig. 2b. The absence of any imaginary phonon modes indicates the dynamical stability of the structure. To verify the theoretical prediction, we then carefully synthesized the TaFeSb compound by the ball-milling and hot-pressing method. Rietveld refinement for the XRD pattern of the prepared specimen indicates that the single-phase $\mathrm{TaFeSb}$ with half-Heusler structure was successfully synthesized. The obtained lattice parameter by Rietveld refinement is $\sim 0.5938$ $\mathrm{nm}$ (Fig. 2c). The selected area electron diffraction (SAED) pattern for the prepared specimen viewed along the [111] direction is shown in Fig. 2d. The three-fold symmetry of the $F \overline{4} 3 \mathrm{~m}$ cubic structure along the [111] direction is well demonstrated by the SAED pattern and the corresponding atomicresolution image (as shown in Supplementary Fig. 1). The lattice parameter $(\sim 0.59 \mathrm{~nm})$ observed by STEM is consistent with the XRD results.

Electronic properties of TaFeSb-based half-Heuslers. Optimization of the thermoelectric performance of TaFeSb-based halfHeuslers was realized by tuning the carrier concentration via Ti-doping. In our work, $\mathrm{Ta}_{1-x} \mathrm{Ti}_{x} \mathrm{FeSb}(x=0,0.02,0.04,0.06$, $0.08,0.10,0.12,0.14,0.16$, and 0.18 ) samples were prepared by the ball-milling and hot-pressing method. All of the samples are highly dense with an average grain size of $\sim 250 \mathrm{~nm}$ (as shown in Supplementary Fig. 2) and they are single-phase with high crystallinity (as shown in Supplementary Fig. 3). For simplicity, the thermoelectric properties of only some of the $\mathrm{Ta}_{1-x} \mathrm{Ti}_{x} \mathrm{FeSb}$ specimens are shown in Fig. 3, while the complete results can be found in Supplementary Fig. 4. As shown in Fig. 3a, the electrical conductivity of the pristine TaFeSb is very low but it increases markedly with Ti-doping. Enhancement of the electrical conductivity should be attributed to the substantially increased hole concentration, as shown in Supplementary Fig. 5. It is $\sim 5.26 \times 10^{20} \mathrm{~cm}^{-3}$ for $\mathrm{Ta}_{0.98} \mathrm{Ti}_{0.02} \mathrm{FeSb}$ and $\sim 2.03 \times 10^{21} \mathrm{~cm}^{-3}$ for $\mathrm{Ta}_{0.84} \mathrm{Ti}_{0.16} \mathrm{FeSb}$. This indicates that $\mathrm{Ti}$ is a very efficient dopant for supplying a high concentration of holes to $\mathrm{TaFeSb}$, which is similar to the case of Ti-doping in $\mathrm{NbFeSb}^{24,25}$. Due to the increased hole concentration, the Seebeck coefficient of $\mathrm{Ta}_{1-x} \mathrm{Ti}_{x} \mathrm{FeSb}$ (with the exception of $\mathrm{TaFeSb}$ ) is reduced gradually with increasing $\mathrm{Ti}$ concentration, as shown in Fig. 3b. It should be noted that the undoped $\mathrm{TaFeSb}$ is fully $\mathrm{p}$-type over the whole temperature range and its Seebeck coefficient increases with temperature without showing any bipolar conduction despite its low hole concentration. This should be ascribed to the relatively large band gap of $\sim 0.53 \mathrm{eV}$ for TaFeSb, which is even larger than that of NbFeSb $(\sim 0.41 \mathrm{eV})$, as shown in Supplementary Fig. 6. Optimization of the holeconcentration substantially improves the power factor of TaFeSb-based
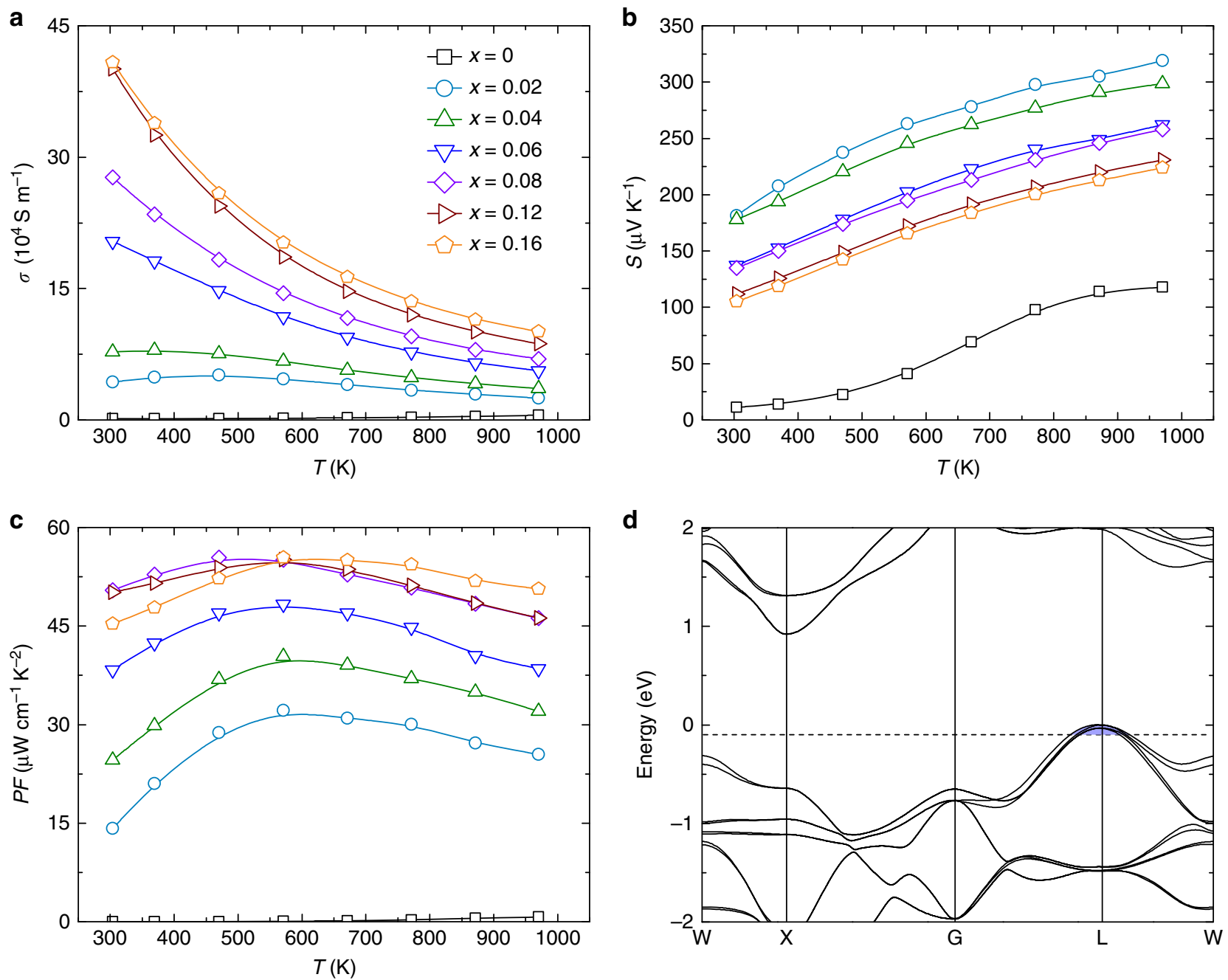

Fig. 3 Electronic properties of TaFeSb-based half-Heuslers. Temperature-dependent a electrical conductivity, b Seebeck coefficient, and c power factor of $\mathrm{Ta}_{1-x} \mathrm{Ti}_{x} \mathrm{FeSb}(x=0,0.02,0.04,0.06,0.08,0.12$, and 0.16). d Band structure of TaFeSb 
half-Heuslers, as shown in Fig. 3c. The room-temperature power factor is $\sim 45 \mu \mathrm{W} \mathrm{cm} \mathrm{K}^{-1} \mathrm{~K}^{-2}$ for $\mathrm{Ta}_{0.84} \mathrm{Ti}_{0.16} \mathrm{FeSb}$ and its peak power factor can be as large as $\sim 56 \mu \mathrm{W} \mathrm{cm}{ }^{-1} \mathrm{~K}^{-2}$, thus leading to its high average power factor of $\sim 52 \mu \mathrm{W} \mathrm{cm}{ }^{-1} \mathrm{~K}^{-2}$ in the temperature range between 300 and $973 \mathrm{~K}$. Such a high power factor outperforms most of the other state-of-the-art half-Heusler compounds, e.g., (Ti/Zr/Hf) $\mathrm{CoSb}^{26-28,} \quad(\mathrm{Ti} / \mathrm{Zr} / \mathrm{Hf}) \mathrm{NiSn}^{29-33}$, $\mathrm{ZrNiPb}^{34}$, and $\mathrm{NbCoSn}^{35,36}$. The origin of the high electronic thermoelectric performance of TaFeSb-based half-Heuslers can be partially ascribed to the unique electronic band structure, as shown in Fig. $3 \mathrm{~d}$. The valence band maximum locates at the Lpoint (with a carrier pocket degeneracy of four), where two valence bands (with an orbital degeneracy of two) effectively converge and thus leading to a very high valley degeneracy $\left(N_{\mathrm{v}}\right)$ of eight (as shown in Supplementary Fig. 7). The thermoelectric figure of merit $Z T$ is closely correlated with the materials' parameter $B$, where $B \propto N_{\mathrm{V}} \mu\left(m^{*}\right)^{3 / 2} / \kappa_{\text {lat }}$ ( $\mu$ is the mobility and $m^{*}$ is the effective mass) ${ }^{1,37}$. Therefore, the high band degeneracy of $\mathrm{TaFeSb}$ is highly beneficial for realizing the large power factor.

Thermal conductivities of TaFeSb-based half-Heuslers. Comparison of the thermal conductivity between the undoped $\mathrm{NbFeSb}$ and TaFeSb is shown in Supplementary Fig. 8. The roomtemperature thermal conductivity is $\sim 14 \mathrm{~W} \mathrm{~m}^{-1} \mathrm{~K}^{-1}$ for $\mathrm{NbFeSb}$ and it is only $\sim 8.8 \mathrm{~W} \mathrm{~m}^{-1} \mathrm{~K}^{-1}$ for TaFeSb. To understand the difference in lattice thermal conductivity, phonon dispersion relations for $\mathrm{NbFeSb}$ and $\mathrm{TaFeSb}$ were calculated as shown in Supplementary Fig. 9. TaFeSb has overall lower phonon frequencies than those of NbFeSb. The acoustic phonon velocity is lower in $\mathrm{TaFeSb}$ compared to NbFeSb. Usually, the phonon velocity can simply be approximated by the low frequency sound velocity ${ }^{38}$. The measured sound velocity is $2907 \mathrm{~m} \mathrm{~s}^{-1}$ for TaFeSb but is $3473 \mathrm{~m} \mathrm{~s}^{-1}$ for $\mathrm{NbFeSb}$ (as shown in Supplementary Fig. 10). According to the kinetic theory, the lattice thermal conductivity can be expressed as $\kappa_{\text {lat }}=\frac{1}{3} C_{\mathrm{v}} v_{\mathrm{ph}} l$, where $C_{\mathrm{v}}$ is the heat capacity per unit volume, $v_{\mathrm{ph}}$ is the phonon velocity, and $l$ is the phonon mean free path ${ }^{39}$. Therefore, the lower phonon velocity of $\mathrm{TaFeSb}$ can account for its lower thermal conductivity compared to $\mathrm{NbFeSb}$.

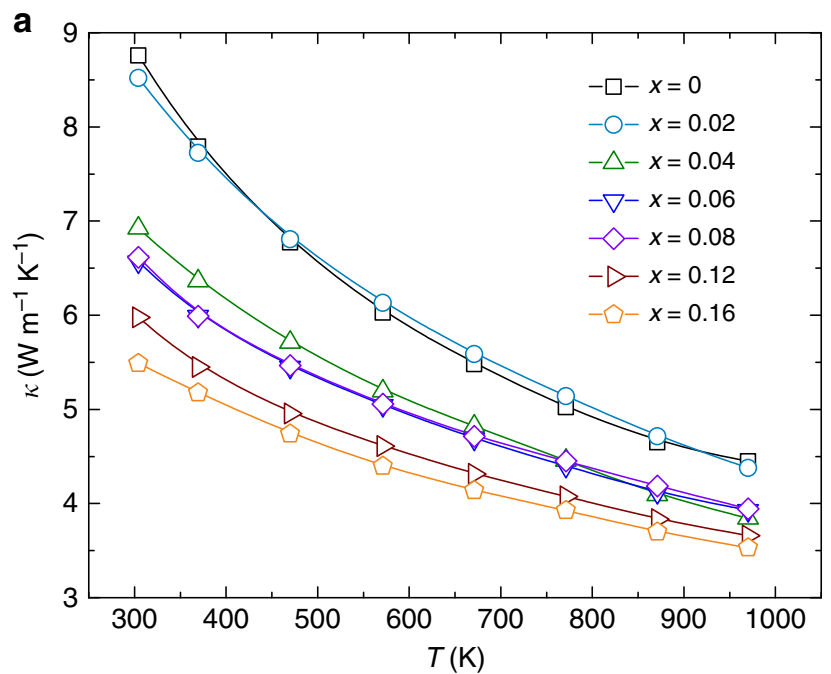

Although the electrical conductivity of TaFeSb has been substantially increased by Ti-doping (which effectively increases the electronic thermal conductivity via the Wiedemann-Franz law $\kappa_{\text {ele }}=L \sigma T$, where $L$ is the Lorenz constant), the total thermal conductivity of $\mathrm{Ta}_{1-x} \mathrm{Ti}_{x} \mathrm{FeSb}$ demonstrates a noticeable reduction with increasing $\mathrm{Ti}$ concentration (as shown in Fig. 4a). The composition-dependent lattice thermal conductivity of $\mathrm{Ta}_{1}$ ${ }_{x} \mathrm{Ti}_{x} \mathrm{FeSb}$ is calculated and shown in Fig. $4 \mathrm{~b}$. It demonstrates a monotonic reduction with respect to Ti concentration. The roomtemperature lattice thermal conductivity is $\sim 8.8 \mathrm{~W} \mathrm{~m}^{-1} \mathrm{~K}^{-1}$ for TaFeSb but is only $\sim 3.1 \mathrm{~W} \mathrm{~m}^{-1} \mathrm{~K}^{-1}$ for $\mathrm{Ta}_{0.84} \mathrm{Ti}_{0.16} \mathrm{FeSb}$, a reduction of $\sim 65 \%$. Such a significant phonon scattering should be ascribed mainly to the presence of point defects induced by $\mathrm{Ti}$ doping at the Ta site. The substantial difference in atomic mass between $\mathrm{Ti}(\sim 47.87)$ and $\mathrm{Ta}(\sim 180.95)$ leads to the strong scattering that effectively disrupts the phonon propagation. By alloying the $\mathrm{Ta}_{0.84} \mathrm{Ti}_{0.16} \mathrm{FeSb}$ composition with VFeSb, the lattice thermal conductivity can be further reduced, as shown in Fig. 4c and Supplementary Fig. 11e. The room-temperature lattice thermal conductivity is $\sim 3.1 \mathrm{~W} \mathrm{~m}^{-1} \mathrm{~K}^{-1}$ for $\mathrm{Ta}_{0.84} \mathrm{Ti}_{0.16} \mathrm{FeSb}$ and is only $\sim 2.3 \mathrm{~W} \mathrm{~m}{ }^{-1} \mathrm{~K}^{-1}$ for $\mathrm{Ta}_{0.74} \mathrm{~V}_{0.1} \mathrm{Ti}_{0.16} \mathrm{FeSb}$, a decrease of $\sim 26 \%$.

$Z T$ and energy conversion efficiency of TaFeSb-based halfHeuslers. Benefitting from the simultaneous enhancement in power factor and reduction in thermal conductivity, the thermoelectric figure of merit of TaFeSb-based half-Heuslers can be effectively improved after Ti-doping. The thermoelectric performance of $\mathrm{Ta}_{1-x} \mathrm{Ti}_{x} \mathrm{FeSb}$ increases with the $\mathrm{Ti}$ concentration and $\mathrm{Ta}_{0.84} \mathrm{Ti}_{0.16} \mathrm{FeSb}$ achieves a high peak $Z T$ of $\sim 1.39$ at $973 \mathrm{~K}$ (as shown in Fig. 5a). As mentioned earlier, by alloying $\mathrm{Ta}_{0.84} \mathrm{Ti}_{0.16} \mathrm{FeSb}$ with VFeSb, it is possible to further reduce the thermal conductivity and the power factor remains similar when $\mathrm{V}$ concentration is less than 15 at.\% (as shown in Supplementary Fig. 11c). Therefore, the thermoelectric performance of TaFeSbbased half-Heuslers can be further improved and $T a_{0.74} V_{0.1^{-}}$ $\mathrm{Ti}_{0.16} \mathrm{FeSb}$ achieves a peak $Z T$ of $\sim 1.52$ at $973 \mathrm{~K}$. To demonstrate the repeatability of our results, $\mathrm{Ta}_{0.74} \mathrm{~V}_{0.1} \mathrm{Ti}_{0.16} \mathrm{FeSb}$ has been prepared in five different batches and the thermoelectric properties are quite similar (as shown in Supplementary Fig. 12).

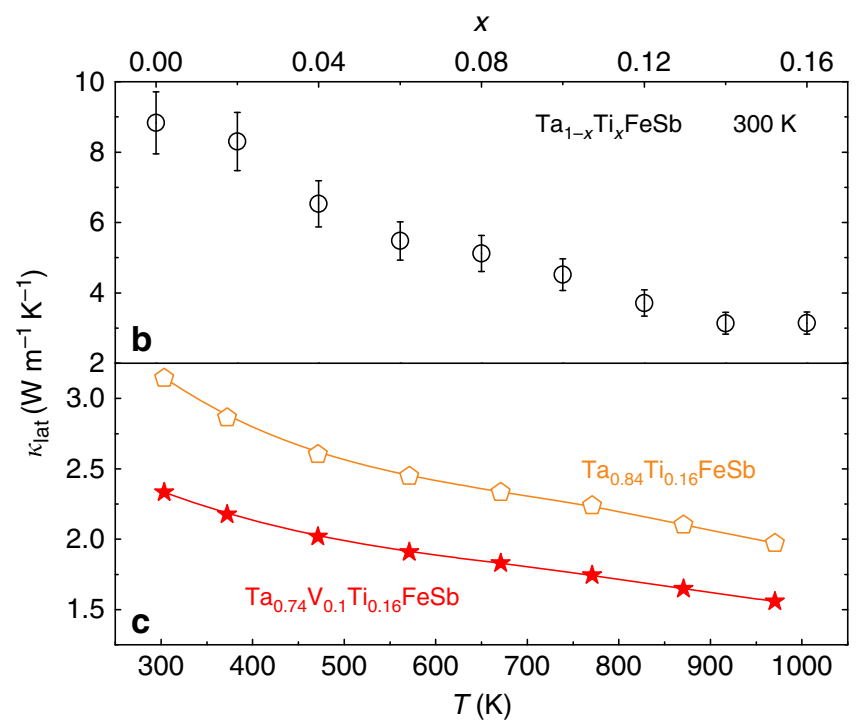

Fig. 4 Thermal conductivity of TaFeSb-based half-Heuslers. a Temperature-dependent thermal conductivity of Ta ${ }_{1-x} \mathrm{Ti}_{x} \mathrm{FeSb}_{1}$, b composition-dependent

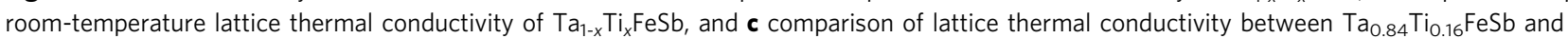
$\mathrm{Ta}_{0.74} \mathrm{~V}_{0.1} \mathrm{Ti}_{0.16} \mathrm{FeSb}$ 
a
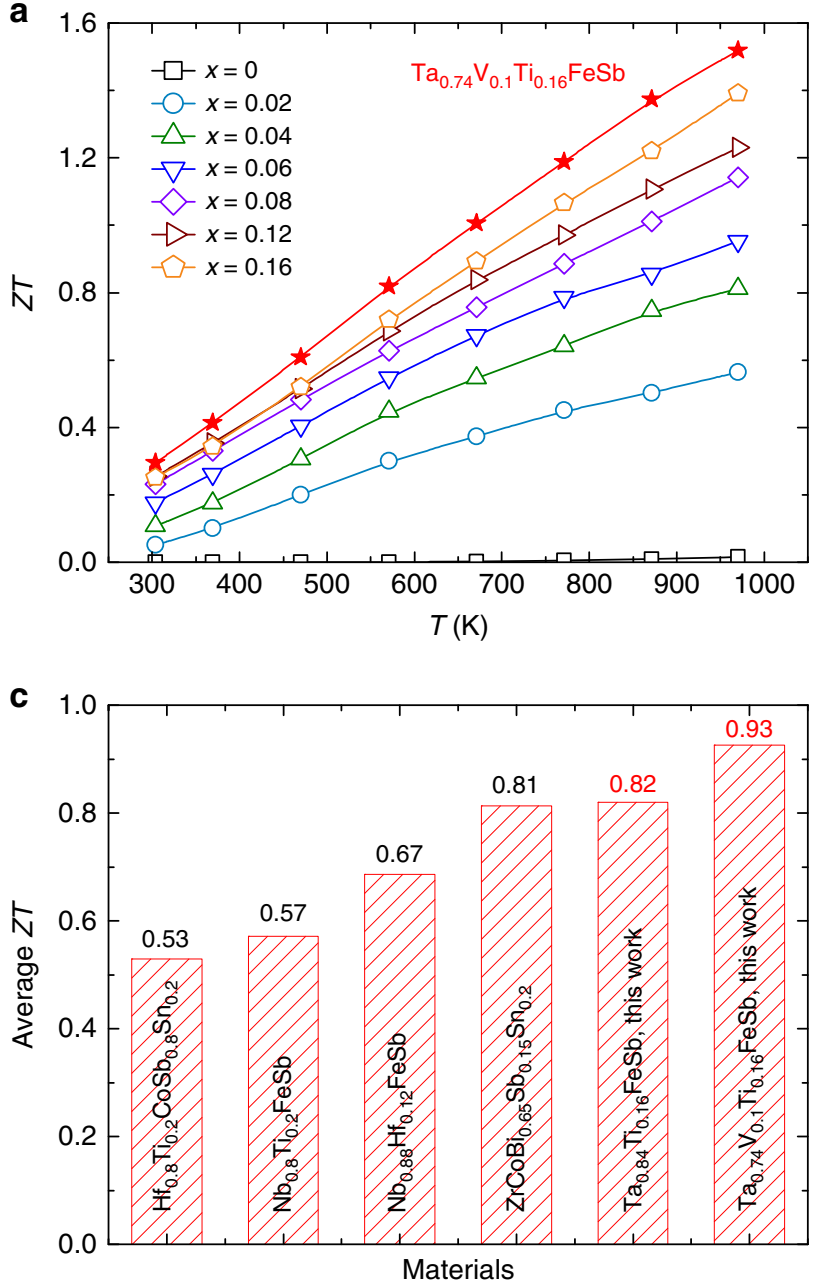

b

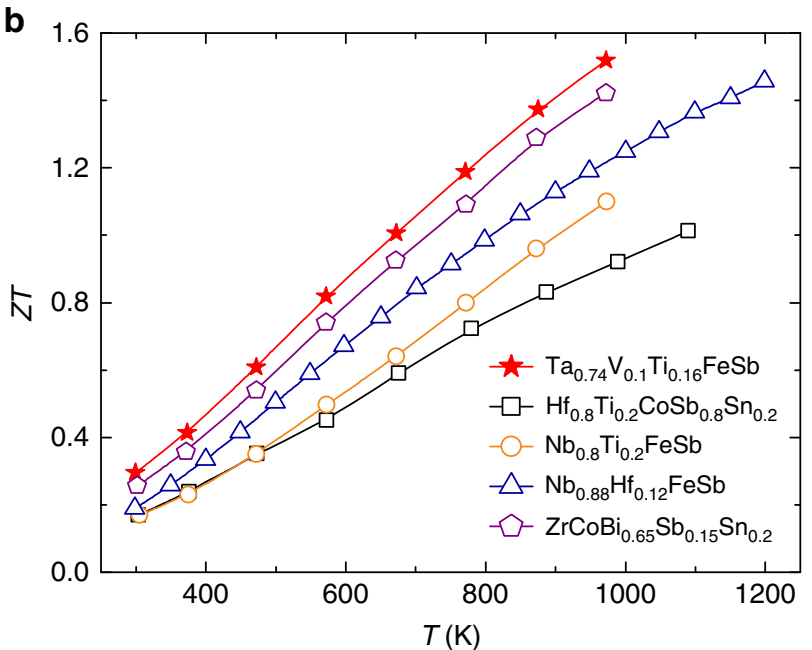

d

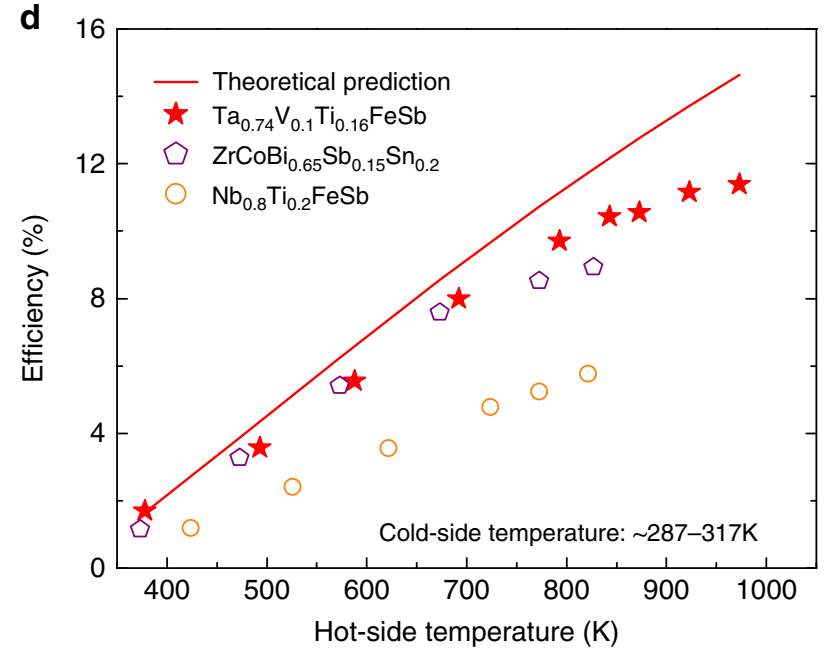

Fig. 5 Dimensionless thermoelectric figure of merit of TaFeSb-based half-Heuslers. a Temperature-dependent $Z T$ of $T a_{1-x} T i_{x} F e S b$, $\mathbf{b}$ comparison of the $Z T$ between $\mathrm{Ta}_{0.74} \mathrm{~V}_{0.1} \mathrm{Ti}_{0.16} \mathrm{FeSb}$ and the other state-of-the-art p-type half-Heuslers ${ }^{24-27}$, c comparison of the average $Z T$ (in the range between 300 and 973 $\mathrm{K}$ ) between TaFeSb-based half-Heuslers and the other state-of-the-art p-type half-Heuslers, and $\mathbf{d}$ the measured hot-side temperature-dependent heat-toelectricity conversion efficiency of $\mathrm{Ta}_{0.74} \mathrm{~V}_{0.1} \mathrm{Ti}_{0.16} \mathrm{FeSb}$ as compared with state-of-the-art p-type half-Heuslers $\mathrm{ZrCoBi}_{0.65} \mathrm{Sb}_{0.15} \mathrm{Sn}_{0.2}{ }^{40}$ and $\mathrm{Nb}_{0.8} \mathrm{Ti}_{0.2} \mathrm{FeSb}^{25}$. The cold-side temperature is fixed at $300 \mathrm{~K}$ for the calculation of efficiency (solid line)

The thermal stability of $\mathrm{Ta}_{0.74} \mathrm{~V}_{0.1} \mathrm{Ti}_{0.16} \mathrm{FeSb}$ was further characterized by repeatedly measuring the same specimen five times and the thermoelectric properties are also comparable (as shown in Supplementary Fig. 13). This indicates that the high thermoelectric performance of $\mathrm{Ta}_{0.74} \mathrm{~V}_{0.1} \mathrm{Ti}_{0.16} \mathrm{FeSb}$ is very stable and repeatable. Comparison of the thermoelectric performance between the TaFeSb-based half-Heuslers with the other p-type state-of-the-art half-Heusler compounds ${ }^{24-27}$ is shown in Fig. 5b. Clearly, $\mathrm{Ta}_{0.74} \mathrm{~V}_{0.1} \mathrm{Ti}_{0.16} \mathrm{FeSb}$ outperforms all of the other halfHeuslers over the whole temperature range between 300 and 973 $\mathrm{K}$. The average $Z T$ is further calculated by the integration method (with a fourth-order polynomial for fitting the $Z T$ curve) between 300 and $973 \mathrm{~K}$ and comparison of the average $Z T$ is shown in Fig. $5 \mathrm{c}$. The average $Z T \mathrm{~s}$ are $\sim 0.53$ for $\mathrm{Hf}_{0.8} \mathrm{Ti}_{0.2} \mathrm{CoSb}_{0.8} \mathrm{Sn}_{0.2}{ }^{26}$, $\sim 0.57$ for $\mathrm{Nb}_{0.8} \mathrm{Ti}_{0.2} \mathrm{FeSb}^{25}, \sim 0.67$ for $\mathrm{Nb}_{0.88} \mathrm{Hf}_{0.12} \mathrm{FeSb}^{24}$, and $\sim 0.81$ for $\mathrm{ZrCoBi}_{0.65} \mathrm{Sb}_{0.15} \mathrm{Sn}_{0.2}{ }^{40}$, all of which are lower than $\sim 0.82$ for $\mathrm{Ta}_{0.84} \mathrm{Ti}_{0.16} \mathrm{FeSb}$ and $\sim 0.93$ for $\mathrm{Ta}_{0.74} \mathrm{~V}_{0.1} \mathrm{Ti}_{0.16} \mathrm{FeSb}$. The high thermoelectric performance of $\mathrm{Ta}_{0.74} \mathrm{~V}_{0.1} \mathrm{Ti}_{0.16} \mathrm{FeSb}$ was further verified by the heat-to-electricity conversion efficiency measurement. As shown in Fig. 5d, the measured peak efficiency of $\mathrm{Ta}_{0.74} \mathrm{~V}_{0.1} \mathrm{Ti}_{0.16} \mathrm{FeSb}$ can be as large as $\sim 11.4 \%$ at the coldside temperature of $317 \mathrm{~K}$ and hot-side temperature of $973 \mathrm{~K}$.
This is a record high efficiency for a single leg based on halfHeusler thermoelectric materials. Our results demonstrate that the TaFeSb-based half-Heuslers are quite promising for thermoelectric power generation.

\section{Discussion}

In summary, thermodynamic stability analysis was performed for the $\mathrm{V}^{1}$-VIII- $\mathrm{V}^{2}$ family (with $\mathrm{V}^{1}=\mathrm{V}, \mathrm{Nb}$, and $\mathrm{Ta}$; $\mathrm{VIII}=\mathrm{Fe}$, $\mathrm{Ru}$, and Os; and $\mathrm{V}^{2}=\mathrm{As}$, Sb, and Bi) of half-Heuslers. We predicted that six compounds (VRuAs, NbRuAs, TaRuAs, TaFeSb, $\mathrm{NbOsSb}$, and TaOsSb) in this family should be stable. To verify this prediction, the TaFeSb-based half-Heuslers were synthesized and their thermoelectric performance was optimized. Our results indicated that $\mathrm{Ta}_{0.74} \mathrm{~V}_{0.1} \mathrm{Ti}_{0.16} \mathrm{FeSb}$ can achieve a peak $Z T$ of $\sim 1.52$ at $973 \mathrm{~K}$, outperforming all of the other state-of-theart p-type half-Heusler materials. More importantly, the heat-toelectricity conversion efficiency was also measured for the TaFeSb-based half-Heuslers and the maximum efficiency can be as large as $\sim 11.4 \%$ at the cold-side temperature of $317 \mathrm{~K}$ and hot-side temperature of $973 \mathrm{~K}$. Our work demonstrated that the TaFeSb-based half-Heuslers are quite promising for thermoelectric power generation. 


\section{Methods}

Synthesis. TaFeSb-based half-Heuslers were prepared by a two-step ball-milling and hot-pressing method. In the first step, Ta turnings (99.98\%; Atlantic Metals \& Alloys) and Sb ingots (99.999\%; Alfa Aesar) were loaded into a stainless-steel jar under an argon atmosphere in a glove box and ball-milled for $15 \mathrm{~h}$. Afterward, Fe granules (99.8\%; Alfa Aesar), Ti granules (99.99\%; Alfa Aesar), and V pieces (99.7\%; Alfa Aesar) were added for another $20 \mathrm{~h}$ ball milling. The ball-milling process was conducted using a SPEX $8000 \mathrm{M}$ Mixer/Mill. The ball-milled powders were compacted to a disk by a direct-current-induced hot-pressing at about $1123 \mathrm{~K}$ for $2 \mathrm{~min}$ and under the pressure of $\sim 80 \mathrm{MPa}$

Thermoelectric properties measurement. The Seebeck coefficient and electrical conductivity were simultaneously obtained by a commercial (ZEM-3, ULVAC) system in helium atmosphere. The thermal conductivity $\kappa=D C_{\mathrm{p}} \rho$ was calculated from the thermal diffusivity $D$ (LFA 457 , Netzsch), specific heat $C_{p}$ (DSC $404 \mathrm{C}$; Netzsch), and mass density $\rho$ (Archimedes' kit). Hall carrier concentrations $\left(n_{\mathrm{H}}\right.$ were measured using a commercial system (PPMS Dynacool, Quantum Design) with a magnetic field of $\pm 3 \mathrm{~T}$ and an electrical current of $8 \mathrm{~mA}$.

Efficiency measurement. The thermoelectric material (referenced as leg in the following discussion) was polished to the size of $2.6 \times 2.7 \mathrm{~mm}^{2}$ in cross-section and $\sim 11 \mathrm{~mm}$ in length. The cold-side temperature was maintained at around $287-317 \mathrm{~K}$ by water circulation. The experiments were conducted under high vacuum (below $10^{-6}$ mbar) to reduce the parasitic conduction and convection losses. To measure conversion efficiency $(\eta)$, the input power from the hot side $\left(Q_{\text {in }}\right)$ and the generated power $(P)$ from the thermoelectric leg were measured at the same time. The direct measurement of $Q_{\text {in }}$ is a greatly challenging due to the heavy heat loss at high temperature. According to Fourier's law, a bulk polycrystalline graphite with measured geometry and thermal conductivity was placed below the cold-side to measure the heat flow out of the cold-side $\left(Q_{\text {out }}\right)$. The thermal conductivity of the bulk polycrystalline graphite was confirmed by the method described above in the discussion on thermoelectric properties measurement. In order to measure temperature differences of the leg and graphite bulk, K-type thermocouples were embedded at the interfaces. It should be noted that the hot-side temperature of graphite can be regarded as the cold-side temperature of the leg if the setup is working under a large pressure. The total $Q_{\text {in }}$ equals the sum of $Q_{\text {out }}, P$, and radiation loss from the leg $\left(Q_{\mathrm{rad}}\right)$. Therefore, the conversion efficiency $(\eta)$ can be written as the following

$$
\eta=\frac{P}{Q_{\text {in }}}=\frac{P}{Q_{\text {out }}+P+Q_{\text {rad }}} .
$$

Since $Q_{\text {rad }}$ cannot be directly measured, in real measurement $Q_{\text {in }}$ is composed of $Q_{\text {out }}$ and $P$, which leads to the measurement error of $\eta$. By tuning the current in the circuit, a series of $Q_{\text {in }}, P$ can be measured at the same time. Therefore, both maximum $\eta$ and $P$ can be found. The estimated radiation loss based on the finite difference method is about $1-6 \%$ of the total heat flow in.

For $p$-type thermoelectrics, the measured open circuit voltage should be smaller since the Seebeck coefficient of copper is a positive number, which can be given by a following empirical function ${ }^{41}$

$$
\begin{gathered}
S_{\mathrm{Cu}}(T)=0.041 T\left[\exp \left(-\frac{T}{93}\right)+0.123-\frac{0.442}{1+(T / 172.4)^{3}}\right]+0.804, \\
70 \mathrm{~K}<T<1000 \mathrm{~K} .
\end{gathered}
$$

The Seebeck coefficient of copper is $\sim 6 \mu \mathrm{V} \mathrm{K}-1$ at $973 \mathrm{~K}$, and the measured output power at $973 \mathrm{~K}$ is underestimated by about $5-6 \%$.

Microstructural characterization. Phase identification was carried out by X-ray diffraction (XRD) on a PANalytical multipurpose diffractometer with an X'Celerator detector (PANalytical X'Pert Pro). The morphology and microstructures were characterized by a field emission scanning electron microscope (FESEM, LEO 1525). Selected area electron diffraction pattern and HAADF-STEM images were obtained by a JEM-ARM 200F TEM operated at $200 \mathrm{kV}$.

Bandgap measurement. Fourier transform infrared spectroscopy (FTIR) was performed to derive the optical band gap based on the Kramers-Kronig analysis of the reflectance. The FTIR was conducted using a Nicolet iS50 FT-IR spectrometer with a Spectra-Tech model 500 series variable angle specular reflectance accessory at room temperature.

Sound velocity measurement. Sound velocity measurements were carried out using a RITEC Advanced Ultrasonic Measurement System RAM-5000. The system realizes the pulse-echo method of time propagation measurements with an accuracy of about $10^{-3} \mu$ s. To generate longitudinal (L) and shear (S) ultrasonic bulk waves, Olympus transducers V129-RM $(10 \mathrm{MHz})$ and V157-RM $(5 \mathrm{MHz})$ were used. Propylene glycol and SWC (both from Olympus) were used as couplant materials for the L and S modes, respectively. Thickness measurements were carried out using a Mitutoyo ID-HO530 device. All data were obtained at $300 \mathrm{~K}$.

Theoretical calculations. We searched for stable $\mathrm{V}^{1}-\mathrm{VIII}-\mathrm{V}^{2}$ half-Heusler crystal structures up to 18 atoms per unit cell using the global optimization particle swarm method as implemented in the CALYPSO code ${ }^{42,43}$. At least 2000 structures were calculated during each search. The key feature of the CALYPSO method is the ability to rapidly identify ground-state structures with knowledge only of the chemical composition. Details of the algorithm and applications have been discussed elsewhere ${ }^{44-46}$. The first-principles calculations of the formation energy were performed using the projector-augmented wave (PAW) method as implemented in the VASP code ${ }^{47,48}$. The electronic structures were obtained with the general potential linearized augmented plane wave (LAPW) method as implemented in the WIEN2k code ${ }^{49}$. This enabled us to realize structure optimization and hybrid functional calculations using VASP, which is time consuming and has the potential compatibility issue (with CALYPSO) in WIEN2K, as well as allowing the convenience of the later transport calculations using WIEN2K. The Perdew, Burke, and Ernzerhof (PBE-GGA) type functional was used for the structural optimization within VASP ${ }^{50}$. Because of the sensitivity of thermodynamic stability, the $3 p^{6} 3 d^{3} 4 s^{2}(\mathrm{~V}), 4 p^{6} 4 d^{4} 5 s^{1}(\mathrm{Nb}), 5 p^{6} 5 d^{3} 6 s^{2}(\mathrm{Ta}), 3 p^{6} 3 d^{6} 4 s^{2}(\mathrm{Fe}), 4 p^{6} 4 d^{7} 5 s^{1}(\mathrm{Ru})$, $5 p^{6} 5 d^{6} 6 s^{2}(\mathrm{Os}), 4 s^{2} 4 p^{3}(\mathrm{As}), 5 s^{2} 5 p^{3}(\mathrm{Sb})$, and $5 d^{10} 6 s^{2} 6 p^{3}(\mathrm{Bi})$ states were treated as valence electrons of PAW pseudopotentials. These were used with kinetic energy cutoffs of $520 \mathrm{eV}$. We conducted tests to ensure that this was adequate. The k-poin meshes for sampling the Brillouin zone were at a grid spacing of $2 \pi \times 0.032 \AA$ or better, including the hybrid functional calculations. Harmonic phonons were obtained with real space supercells using the PHONOPY code ${ }^{51}$.

Band structure and density of states (DOS) calculations were conducted by using the modified Becke-Johnson $(\mathrm{mBJ})$ potential of Tran and Blaha ${ }^{52,53}$. This $\mathrm{mBJ}$ potential gives band gaps in agreement with experiment for a wide variety of simple semiconductors and insulators ${ }^{54,55}$. In the present case, our calculated band gap of $0.9 \mathrm{eV}$ is larger than the experimental value, perhaps due to site disorder in the half-Heusler structure. We also cross-checked the band gaps using the hybrid Heyd-Scuseria-Ernzerhof (HSE06) functional ${ }^{56}$, which also supports a larger band gap. Spin-orbit coupling (SOC) was included in electronic structure calculations, as the spin-orbit splitting may be very large for heavy elements. We used LAPW sphere radii of $2.5 \mathrm{bohr}$ for TaFeSb. A basis set cutoff, $K_{\max }$, determined by the criterion $R_{\min } K_{\max }=9.0$, was used. Here, $R_{\min }$ denotes the minimum atomic radius of elements.

\section{Data availability}

The data that support the findings of this study are available from the corresponding author upon reasonable request.

Received: 6 July 2018 Accepted: 28 November 2018 Published online: 17 January 2019

\section{References}

1. DiSalvo, F. J. Thermoelectric cooling and power generation. Science 285, 703-706 (1999).

2. Bell, L. E. Cooling, heating, generating power, and recovering waste heat with thermoelectric systems. Science 321, 1457-1461 (2008).

3. Zhu, T. J. et al. Compromise and synergy in high-efficiency thermoelectric materials. Adv. Mater. 29, 1605884 (2017).

4. He, J. \& Tritt, T. M. Advances in thermoelectric materials research: looking back and moving forward. Science 357, eaak9997 (2017).

5. Liu, W. S. et al. New trends, strategies and opportunities in thermoelectric materials: a perspective. Mater. Today Phys. 1, 50-60 (2017).

6. Liu, Z., Mao, J., Sui, J. \& Ren, Z. F. High thermoelectric performance of a-MgAgSb for power generation. Energy Environ. Sci. 11, 23-44 (2018).

7. Mao, J. et al. Advances in thermoelectrics. Adv. Phys. 67, 69-147 (2018)

8. Ioffe, A. F. Semiconductor thermoelements and thermoelectric cooling (Infosearch, London, 1957).

9. Heremans, J. P. et al. Enhancement of thermoelectric efficiency in $\mathrm{PbTe}$ by distortion of the electronic density of states. Science 321, 554-557 (2008)

10. Pei, Y. Z. et al. Convergence of electronic bands for high performance bulk thermoelectrics. Nature 473, 66-69 (2011)

11. Liu, W. et al. Convergence of conduction bands as a means of enhancing thermoelectric performance of n-type $\mathrm{Mg}_{2} \mathrm{Si}_{1-\mathrm{x}} \mathrm{Sn}_{\mathrm{x}}$ solid solutions. Phys. Rev. Lett. 108, 166601 (2012).

12. Zhao, L. D. et al. Ultralow thermal conductivity and high thermoelectric figure of merit in SnSe crystals. Nature 508, 373-377 (2014).

13. Mao, J. et al. Manipulation of ionized impurity scattering for achieving high thermoelectric performance in n-type $\mathrm{Mg}_{3} \mathrm{Sb}_{2}$-based materials. Proc. Natl Acad. Sci. USA 114, 10548-10553 (2017). 
14. Chang, C. et al. 3D charge and 2D phonon transports leading to high out-ofplane ZT in n-type SnSe crystals. Science 360, 778-783 (2018).

15. Curtarolo, S. et al. The high-throughput highway to computational materials design. Nat. Mater. 12, 191-201 (2013).

16. Zhou, J. et al. Large thermoelectric power factor from crystal symmetryprotected non-bonding orbital in half-Heuslers. Nat. Commun. 9, 1721 (2018).

17. Gautier, R. et al. Prediction and accelerated laboratory discovery of previously unknown 18-electron ABX compounds. Nat. Chem. 7, 308-316 (2015).

18. Carrete, J. et al. Finding unprecedentedly low-thermal-conductivity halfHeusler semiconductors via high-throughput materials modeling. Phys. Rev. X 4, 011019 (2014)

19. Zakutayev, A. et al. Theoretical prediction and experimental realization of new stable inorganic materials using the inverse design approach. J. Am. Chem. Soc. 135, 10048-10054 (2013).

20. Zunger, A. Inverse design in search of materials with target functionalities. Nat. Rev. Chem. 2, 0121 (2018).

21. Bhattacharya, S. \& Madsen, G. K. A novel p-type half-Heusler from highthroughput transport and defect calculations. J. Mater. Chem. C. 4, 11261-11268 (2016).

22. Massalski, T. B. Binary alloy phase diagrams. ASM Int. 3, 2874 (1992).

23. Failamani, F. et al. Constitution of the systems $\{\mathrm{V}, \mathrm{Nb}, \mathrm{Ta}\}-\mathrm{Sb}$ and physical properties of di-antimonides $\{\mathrm{V}, \mathrm{Nb}, \mathrm{Ta}\} \mathrm{Sb}_{2}$. Intermetallics 65, 94-110 (2015).

24. Fu, C. G. et al. Realizing high figure of merit in heavy-band p-type halfHeusler thermoelectric materials. Nat. Commun. 6, 8144 (2015).

25. He, R. et al. Achieving high power factor and output power density in p-type half-Heuslers $\mathrm{Nb}_{1-\mathrm{x}} \mathrm{Ti}_{\mathrm{x}} \mathrm{FeSb}$. Proc. Natl Acad. Sci. USA 113, 13576-13581 (2016).

26. Yan, X. et al. Stronger phonon scattering by larger differences in atomic mass and size in p-type half-Heuslers $\mathrm{Hf}_{1-\mathrm{x}} \mathrm{Ti}_{\mathrm{x}} \mathrm{CoSb}_{0.8} \mathrm{Sn}_{0.2}$. Energy Environ. Sci. 5, 7543-7548 (2012).

27. Yan, X. et al. Thermoelectric property study of nanostructured p-type halfHeuslers (Hf, Zr, Ti) $\mathrm{CoSb}_{0.8} \mathrm{Sn}_{0.2}$. Adv. Energy Mater. 3, 1195-1200 (2013).

28. He, R. et al. Improved thermoelectric performance of $\mathrm{n}$-type half-Heusler $\mathrm{MCo}_{1-\mathrm{x}} \mathrm{Ni}_{\mathrm{x}} \mathrm{Sb}(\mathrm{M}=\mathrm{Hf}, \mathrm{Zr})$. Mater. Today Phys. 1, 24-30 (2017).

29. Bhattacharya, $\mathrm{S}$. et al. Effect of $\mathrm{Sb}$ doping on the thermoelectric properties of Ti-based half-Heusler compounds, $\mathrm{TiNiSn}_{1-\mathrm{x}} \mathrm{Sb}_{\mathrm{x}}$. Appl. Phys. Lett. 77, 2476-2478 (2000).

30. $\mathrm{Yu}, \mathrm{C}$. et al. High-performance half-Heusler thermoelectric materials $\mathrm{Hf}_{1}$. ${ }_{\mathrm{x}} \mathrm{Zr}_{\mathrm{x}} \mathrm{NiSn}_{1-\mathrm{y}} \mathrm{Sb}_{\mathrm{y}}$ prepared by levitation melting and spark plasma sintering. Acta Mater. 57, 2757-2764 (2009).

31. Joshi, G. et al. Enhancement in thermoelectric figure-of-merit of an n-type half-Heusler compound by the nanocomposite approach. Adv. Energy Mater. 1, 643-647 (2011).

32. Chen, S. et al. Effect of Hf concentration on thermoelectric properties of nanostructured n-type half-Heusler materials $\mathrm{Hf}_{\mathrm{x}} \mathrm{Zr}_{1-\mathrm{x}} \mathrm{NiSn}_{0.99} \mathrm{Sb}_{0.01}$. $A d v$. Energy Mater. 3, 1210-1214 (2013).

33. Rogl, G. et al. (V, Nb)-doped half Heusler alloys based on $\{\mathrm{Ti}, \mathrm{Zr}, \mathrm{Hf}\} \mathrm{NiSn}$ with high ZT. Acta Mater. 131, 336-348 (2017).

34. Mao, J. et al. Thermoelectric properties of n-type $\mathrm{ZrNiPb}$-based half-Heuslers. Chem. Mater. 29, 867-872 (2017).

35. Kimura, Y., Tamura, Y. \& Kita, T. Thermoelectric properties of directionally solidified half-Heusler compound NbCoSn alloys. Appl. Phys. Lett. 92, 2105 (2008).

36. He, R. et al. Enhanced thermoelectric properties of n-type NbCoSn halfHeusler by improving phase purity. APL Mater. 4, 104804 (2016).

37. Wang, H., Pei, Y., LaLonde, A. D. \& Snyder, G. J. in Thermoelectric Nanomaterials (eds. Koumoto, K. \& Mori, T.). (Springer, Berlin, Heidelberg, 2013).

38. Toberer, E. S., Zevalkink, A. \& Snyder, G. J. Phonon engineering through crystal chemistry. J. Mater. Chem. 21, 15843-15852 (2011).

39. Toberer, E. S., Baranowski, L. L. \& Dames, C. Advances in thermal conductivity. Annu. Rev. Mater. Res. 42, 179-209 (2012).

40. Zhu, H. T. et al. Discovery of ZrCoBi-based half-Heuslers with high thermoelectric conversion efficiency. Nat. Commun. 9, 2497 (2018).

41. Burkov, A. et al. Experimental set-up for thermopower and resistivity measurements at 100-1300 K. Meas. Sci. Technol. 12, 264 (2001).

42. Wang, Y., Lv, J., Zhu, L. \& Ma, Y. Crystal structure prediction via particleswarm optimization. Phys. Rev. B 82, 094116 (2010).

43. Wang, Y., Lv, J., Zhu, L. \& Ma, Y. CALYPSO: a method for crystal structure prediction. Comput. Phys. Commun. 183, 2063-2070 (2012).

44. Zhang, X. et al. First-principles structural design of superhard materials. J. Phys. Chem. 138, 114101 (2013).

45. Li, Y. et al. Design of ternary alkaline-earth metal Sn (II) oxides with potential good p-type conductivity. J. Mater. Chem. C. 4, 4592-4599 (2016).

46. Li, Y., Zhang, L. \& Singh, D. J. New stable ternary alkaline-earth metal Pb (II) oxides: $\mathrm{Ca} / \mathrm{Sr} / \mathrm{BaPb}_{2} \mathrm{O}_{3}$ and $\mathrm{BaPbO}_{2}$. Phys. Rev. Mater. 1, 055001 (2017).

47. Kresse, G. \& Furthmüller, J. Self-interaction correction to density functional approximation for many electron systems. Phys. Rev. B 54, 11169 (1996).
48. Kresse, G. \& Joubert, D. From ultrasoft pseudopotentials to the projector augmented-wave method. Phys. Rev. B 59, 1758 (1999).

49. Blaha, P. et al. wien $2 \mathrm{k}$. An augmented plane wave + local orbitals program for calculating crystal properties, (Technische Universität Wien, Vienna, 2001)

50. Perdew, J. P., Burke, K. \& Ernzerhof, M. Generalized gradient approximation made simple. Phys. Rev. Lett. 77, 3865 (1996).

51. Togo, A. \& Tanaka, I. First principles phonon calculations in materials science. Scr. Mater. 108, 1-5 (2015).

52. Tran, F. \& Blaha, P. Accurate band gaps of semiconductors and insulators with a semilocal exchange-correlation potential. Phys. Rev. Lett. 102, 226401 (2009).

53. Koller, D., Tran, F. \& Blaha, P. Merits and limits of the modified BeckeJohnson exchange potential. Phys. Rev. B 83, 195134 (2011).

54. Li, Y., Sun, J. \& Singh, D. J. Infrared absorption and visible transparency in heavily doped p-type $\mathrm{BaSnO}_{3}$. Appl. Phys. Lett. 110, 051904 (2017).

55. Li, S. Y. et al. Optical properties of $\mathrm{Cu}_{2} \mathrm{ZnSn}\left(\mathrm{S}_{\mathrm{x}} \mathrm{Se}_{1-\mathrm{x}}\right)_{4}$ solar absorbers: spectroscopic ellipsometry and ab initio calculations. Appl. Phys. Lett. 110, 021905 (2017).

56. Heyd, J., Scuseria, G. E. \& Ernzerhof, M. Hybrid functionals based on a screened Coulomb potential. J. Phys. Chem. 118, 8207-8215 (2003).

\section{Acknowledgements}

The work is partly supported by the Basic Energy Science Program of the Department of Energy under a grant DE-SC0010831 and the Solid-State Solar-Thermal Energy Conversion Center ( $\left.\mathrm{S}^{3} \mathrm{TEC}\right)$, an Energy Frontier Research Center funded by the U.S. Department of Energy, Office of Science, Office of Basic Energy Sciences under Award Number DE-SC0001299. Y.W. acknowledges the National Natural Science Foundation of China (Grant No. 11474329). Z.W. acknowledges the National Natural Science Foundation of China (Grant No. 51272038) and the National Program on Key Basic Research Project (973 program) (2013CB933301). J.L. acknowledges the National Natural Science Foundation of China (Grant No. 51772186). G.L. acknowledges the National Natural Science Foundation of China (Grant No. 51501159). J.B. acknowledges the Welch Foundation (E-1728).

\section{Author contributions}

H.Z., J.M., and Z.R. designed the research; H.Z., J.M., Y.L., and J.S. performed research; H.Z. and Q.Z. measured the efficiency; Y.W. conducted the TEM characterization; Q.S., J.Z., and Y.F. conducted the first-principles calculation; R.H. and A.S. measured the sound velocity; G.L. performed the Rietveld refinement; T.T. and J.B. conducted the bandgap measurement; Z.L., W.R., Q.S., J.Z., L.Y., Z.W., J.L., K.N., A.S., and G.C. contributed analytical tools; H.Z., J.M., D.J.S., and Z.R. prepared the manuscript; and everyone commented on the manuscript.

\section{Additional information}

Supplementary Information accompanies this paper at https://doi.org/10.1038/s41467 018-08223-5.

Competing interests: The authors declare no competing interests.

Reprints and permission information is available online at http://npg.nature.com/ reprintsandpermissions/

Journal peer review information: Nature Communications thanks the anonymous reviewers for their contribution to the peer review of this work.

Publisher's note: Springer Nature remains neutral with regard to jurisdictional claims in published maps and institutional affiliations.

Open Access This article is licensed under a Creative Commons Attribution 4.0 International License, which permits use, sharing, adaptation, distribution and reproduction in any medium or format, as long as you give appropriate credit to the original author(s) and the source, provide a link to the Creative Commons license, and indicate if changes were made. The images or other third party material in this article are included in the article's Creative Commons license, unless indicated otherwise in a credit line to the material. If material is not included in the article's Creative Commons license and your intended use is not permitted by statutory regulation or exceeds the permitted use, you will need to obtain permission directly from the copyright holder. To view a copy of this license, visit http://creativecommons.org/ licenses/by/4.0/.

(C) The Author(s) 2019 